\title{
Recent Advance in Function Spaces and Their Applications in Fractional Differential Equations
}

\author{
Xinguang Zhang $\left(\mathbb{D},{ }^{1,2,3}\right.$ Lishan Liu $(D), 3$ Yonghong Wu $\left(\mathbb{D},{ }^{2}\right.$ and Liguang Wang ${ }^{3}{ }^{3}$ \\ ${ }^{1}$ School of Mathematical and Informational Sciences, Yantai University, Yantai 264005, Shandong, China \\ ${ }^{2}$ Department of Mathematics and Statistics, Curtin University of Technology, Perth, WA 6845, Australia \\ ${ }^{3}$ School of Mathematical Sciences, Qufu Normal University, Qufu 273165, Shandong, China
}

Correspondence should be addressed to Xinguang Zhang; xinguang.zhang@curtin.edu.au

Received 23 April 2019; Accepted 23 April 2019; Published 7 May 2019

Copyright (C) 2019 Xinguang Zhang et al. This is an open access article distributed under the Creative Commons Attribution License, which permits unrestricted use, distribution, and reproduction in any medium, provided the original work is properly cited.

Fractional calculus is a new branch of analytical mathematics which provides useful tools to model many physical and biological phenomena and optimal control of complex processes with memory effects. Therefore, new advancement of fractional calculus theory will greatly promote the development of function space theory, functional theory, and mathematical physics as well as their applications in differential and integral equations.

This special issue mainly focuses on the latest achievements and recent development of fractional calculus in the nonlinear analysis, optimal control, computational methods, space theory, and applications for solving various fractional differential equations; it contains 46 papers selected through a rigorous peer-reviewed process. These papers almost cover most of directions and applications in fractional calculus.

In what follows, we briefly review the highlights and main contributions of each paper.

In the paper titled "Existence Results for a Class of Semilinear Fractional Partial Differential Equations with Delay in Banach Spaces", the authors consider the existence and uniqueness of the mild solutions for a class of nonlinear time fractional partial differential equations with delay by using the theory of solution operator and the general Banach contraction mapping principle. What is significant about this paper is that it does not need extra conditions to ensure the contraction constant $0<k<1$.

In the paper titled "Some Remarks on Estimate of MittagLeffler Function", the authors point out the mistakes in the estimation process of Mittag-Leffler function, provide a counterexample, and then propose some sufficient conditions to guarantee that part of the estimate for Mittag-Leffler function is correct. Meanwhile, numerical examples are given to illustrate the validity of the two newly established estimates.

In the paper titled "Differential Harnack Estimates for a Semilinear Parabolic System", the author uses the inequalities to construct classical Harnack estimates by integrating along space-time and then proves some differential Harnack inequalities for positive solutions of a semilinear parabolic system on hyperbolic space.

In the paper titled "Toeplitz Operator and Carleson Measure on Weighted Bloch Spaces", the author considers Toeplitz operator acting on weighted Bloch spaces. Meanwhile, the inclusion map from weighted Bloch spaces into tent space is also investigated.

In the paper titled "Solutions for a Class of Hadamard Fractional Boundary Value Problems with Sign-Changing Nonlinearity", by using fixed point index methods, the authors establish some existence theorems of positive (nontrivial) solutions for a class of Hadamard fractional boundary value problems with sign-changing nonlinearity.

In the paper titled "Option Pricing under the Jump Diffusion and Multifactor Stochastic Processes", the authors extend the Heston model to be a hybrid option pricing model driven by multiscale stochastic volatility and jump diffusion process. In this model the correlation effects have been taken into consideration. For the reason that the combination of multiscale volatility processes and jump diffusion process 
results in a high dimensional differential equation, an efficient finite element method is proposed and the integral term arising from the jump term is absorbed to simplify the problem. The numerical results show an efficient explanation for volatility smirks when one incorporates jumps into both the stock process and the volatility process.

In the paper titled "Positive Solutions for a Higher-Order Semipositone Nonlocal Fractional Differential Equation with Singularities on Both Time and Space Variable", the author was concerned with a class of higher-order semipositone nonlocal Riemann-Liouville fractional differential equations. The existence results of positive solutions are given by GuoKrasnosel'skii fixed point theorem and Schauder's fixed point theorem.

In the paper titled "Two New Geraghty Type Contractions in Gb-Metric Spaces", two Geraghty type contractions are introduced in $G b$-metric spaces, and some fixed point theorems about the contractions are proved. At the end of this article, a theorem about unique solution of an integral function is proved.

In the paper titled "Solution of Hamilton-Jacobi-Bellman Equation in Optimal Reinsurance Strategy under Dynamic VaR Constraint", the authors analyze the optimal reinsurance strategy for insurers with a generalized mean-variance premium principle. The surplus process of the insurer is described by the diffusion model which is an approximation of the classical Cramér-Lundberg model by assuming the dynamic VaR constraints for proportional reinsurance; the closed form expression of the optimal reinsurance strategy and corresponding survival probability under proportional reinsurance are obtained.

In the paper titled "Parameter Estimation for Fractional Diffusion Process with Discrete Observations", the authors deal with the problem of estimating the parameters for fractional diffusion process from discrete observations when the Hurst parameter $H$ is unknown. With combination of several methods, such as the Donsker type approximate formula of fractional Brownian motion, quadratic variation method, and the maximum likelihood approach, the authors give the parameter estimations of the Hurst index, diffusion coefficients, and volatility and then prove their strong consistency. Finally, an extension for generalized fractional diffusion process and further work are briefly discussed.

In the paper titled "Hermite-Hadamard-Fejér Inequalities for Conformable Fractional Integrals via Preinvex Functions", the authors present a Hermite-Hadamard-Fejér inequality for conformable fractional integrals by using symmetric preinvex functions and also establish an identity associated with the right hand side of Hermite-Hadamard inequality for preinvex functions; then by using this identity and preinvexity of functions and some well-known inequalities, several new Hermite-Hadamard type inequalities for conformal fractional integrals were established.

In the paper titled "Existence of Nontrivial Solutions for Fractional Differential Equations with p-Laplacian", by combining the properties of the Green function with some fixed point theorems, the authors consider the existence of nontrivial solutions for fractional equations with $p$-Laplacian operator.
In the paper titled "Fixed Point Theory and Positive Solutions for a Ratio-Dependent Elliptic System", the authors consider a ratio-dependent predator-prey model under zero Dirichlet boundary condition. By using topological degree theory and fixed index theory, the necessary and sufficient conditions for the existence of positive solutions were studied, and by bifurcation theory and energy estimates, the asymptotic behavior analysis of positive solutions was presented.

In the paper titled "Existence of Nontrivial Solutions for Some Second-Order Multipoint Boundary Value Problems", by using fixed point theorems with lattice structure, the existence of negative solution and sign-changing solution for some second-order multipoint boundary value problems is obtained.

In the paper titled "Existence of Uniqueness and Nonexistence Results of Positive Solution for Fractional Differential Equations Integral Boundary Value Problems", the author considered a class of fractional differential equations with conjugate type integral conditions. Both the existence of uniqueness and nonexistence of positive solution are obtained by means of the iterative technique. The interesting point is that the assumption on nonlinearity is closely associated with the spectral radius corresponding to the relevant linear operator.

In the paper titled "New Fixed Point Theorems and Application of Mixed Monotone Mappings in Partially Ordered Metric Spaces", the authors consider the existence of a coupled fixed point for mixed monotone mapping satisfying a new contractive inequality which involves an altering distance function in partially ordered metric spaces. Some uniqueness results for coupled fixed points, as well as the existence of fixed points of mixed monotone operators, are established.

In the paper titled "Limit Cycles and Invariant Curves in a Class of Switching Systems with Degree Four", a class of switching systems which have an invariant conic is investigated. Half attracting invariant conic is found in switching systems. The coexistence of small-amplitude limit cycles, large amplitude limit cycles, and invariant algebraic curves under perturbations of the coefficients of the systems is proved.

In the paper titled "Positive Solutions for a System of Fractional Differential Equations with Two Parameters", the existence of positive solutions in terms of different values of two parameters for a system of conformable-type fractional differential equations with the $\mathrm{p}$-Laplacian operator is obtained via Guo-Krasnosel'skii fixed point theorem.

In the paper titled "Impulsive Fractional Differential Equations with p-Laplacian Operator in Banach Spaces", the authors study a class of boundary value problems with multiple point boundary conditions of impulsive p-Laplacian operator fractional differential equations. The sufficient conditions for the existence of solutions in Banach spaces are established by using the Kuratowski noncompactness measure and the Sadovskii fixed point theorem. An example is given to demonstrate the main results.

In the paper titled "Separated Boundary Value Problems of Sequential Caputo and Hadamard Fractional Differential 
Equations", the authors discuss the existence and uniqueness of solutions for new classes of separated boundary value problems of Caputo-Hadamard and Hadamard-Caputo sequential fractional differential equations by using standard fixed point theorems. The application of the obtained results with the aid of examples is demonstrated.

In the paper titled "The Existence and Uniqueness of Solutions and Lyapunov-Type Inequality for CFR Fractional Differential Equations", the authors study CFR fractional differential equations with the derivative of order $3<\alpha<$ 4 and prove existence and uniqueness theorems for CFRtype initial value problem. By Green's function and its corresponding maximum value, the Lyapunov-type inequality of corresponding equations is obtained.

The paper titled "Solutions for Integral Boundary Value Problems of Nonlinear Hadamard Fractional Differential Equations" is aimed at establishing some existence theorems of positive (nontrivial) solutions for integral boundary value problems of nonlinear Hadamard fractional differential equations by using fixed point methods.

In the paper titled "The Conjugate Gradient Viscosity Approximation Algorithm for Split Generalized Equilibrium and Variational Inequality Problems", the authors study a kind of conjugate gradient viscosity approximation algorithm for finding a common solution of split generalized equilibrium problem and variational inequality problem. Under mild conditions, the authors prove that the sequence generated by the proposed iterative algorithm converges strongly to the common solution. Some numerical results are illustrated to show the feasibility and efficiency of the proposed algorithm.

In the paper titled "Some Properties for Solutions of Riemann-Liouville Fractional Differential Systems with a Delay", the authors study properties for solutions of Riemann-Liouville fractional differential systems with a delay. Some results on integral inequalities are first presented by Holder inequality. Then by using the obtained inequalities, the properties on solutions for R-L fractional systems with a delay are investigated and upper bound of solutions is obtained. An illustrative example is considered to support the results

In the paper titled "Existence Results for Impulsive Fractional $q$-Difference Equation with Antiperiodic Boundary Conditions", the authors investigate the impulsive fractional $q$-difference equation with antiperiodic conditions. The existence and uniqueness results of solutions are established via the theorem of nonlinear alternative of Leray-Schauder type and the Banach contraction mapping principle. Two examples are given to illustrate the main results.

The paper titled "Existence of Generalized Nash Equilibrium in $n$-Person Noncooperative Games under Incomplete Preference" presents a new method to improve the existence of Nash equilibrium. Based on the incomplete preference corresponding to equivalence class set being a partial order set, the author translates the incomplete preference problems into the partial order problems. Using the famous Zorn lemma, the existence theorems of fixed point for noncontinuous operators in incomplete preference sets are obtained. Finally, the existence of generalized Nash equilibrium is strictly proved in the $n$-person noncooperative games under incomplete preference

In the paper titled "Positive Solutions for Higher Order Nonlocal Fractional Differential Equation with Integral Boundary Conditions", by using the spectral analysis of the relevant linear operator and Gelfand's formula, some properties of the first eigenvalue of a fractional differential equation were obtained; combining fixed point index theorem, sufficient conditions for the existence of positive solutions are established. An example is given to demonstrate the application of the main results.

In the paper titled "Hopf Bifurcation of a Delayed Ecoepidemic Model with Ratio-Dependent Transmission Rate", the authors mainly focus on the effects of the time delay due to the gestation of the predator for a developed delay ecoepidemic model with ratio-dependent transmission rate. Sufficient conditions for local stability and existence of a Hopf bifurcation of the model are derived by regarding the time delay as the bifurcation parameter. Furthermore, properties of the Hopf bifurcation are investigated by using the normal form theory and the center manifold theorem. Finally, numerical simulations are carried out in order to validate the obtained theoretical results

In the paper titled "Uniqueness of Successive Positive Solution for Nonlocal Singular Higher-Order Fractional Differential Equations Involving Arbitrary Derivatives", by means of fixed point theorem on mixed monotone operator, the authors establish the uniqueness of positive solution for some nonlocal singular higher-order fractional differential equations involving arbitrary derivatives. The iterative schemes for approximating unique positive solution are given.

The paper titled "Existence and Nonexistence of Positive Solutions for Mixed Fractional Boundary Value Problem with Parameter and $p$-Laplacian Operator" mainly studies a class of mixed fractional boundary value problem with parameter and $p$-Laplacian operator. Based on the GuoKrasnosel'skii fixed point theorem, results on the existence and nonexistence of positive solutions for the fractional boundary value problem are established. An example is then presented to illustrate the effectiveness of the results

In the paper titled "Existence Results for Generalized Bagley-Torvik Type Fractional Differential Inclusions with Nonlocal Initial Conditions", the authors prove the existence of solutions for the generalized Bagley-Torvik type fractional-order differential inclusions with nonlocal conditions. It allows one to apply the noncompactness measure of Hausdorff, fractional calculus theory and the nonlinear alternative for Kakutani maps fixed point theorem to obtain the existence results under the assumptions that the nonlocal item is compact continuous and Lipschitz continuous and multifunction is compact and Lipschitz, respectively.

In the paper titled "On the Effective Reducibility of a Class of Quasi-Periodic Linear Hamiltonian Systems Close to Constant Coefficients", the authors consider the effective reducibility of a class of quasi-periodic linear Hamiltonian system. Under nonresonant conditions, it is proved that this system can be reduced to low order system and the change of 
variables that perform such a reduction is also quasi-periodic with the same basic frequencies.

In the paper titled "A Note on the Fractional Generalized Higher Order KdV Equation", the author obtains exact solutions to the fractional generalized higher order Korteweg-de Vries equation using the complex method, showing that the applied method is very useful and is practically well suited for the nonlinear differential equations arising in mathematical physics.

In the paper titled "The Eigenvalue Problem for Caputo Type Fractional Differential Equation with Riemann-Stieltjes Integral Boundary Conditions", the authors investigate the eigenvalue problem for Caputo fractional differential equation with Riemann-Stieltjes integral boundary conditions. By using the Guo-Krasnoselskii's fixed point theorem on cone and the properties of the Green's function, some new results on the existence and nonexistence of positive solutions for the fractional differential equation are obtained.

The paper titled "Synchronization of Different Uncertain Fractional-Order Chaotic Systems with External Disturbances via T-S Fuzzy Model" presents an adaptive fuzzy synchronization control strategy for a class of different uncertain fractional-order chaotic/hyperchaotic systems with unknown external disturbances via T-S fuzzy systems, where the parallel distributed compensation technology is provided to design adaptive controller with fractional adaptation laws. T-S fuzzy models are employed to approximate the unknown nonlinear systems and tracking error signals are used to update the parametric estimates. The asymptotic stability of the closed-loop system and the boundedness of the states and parameters are guaranteed by fractional Lyapunov theory. This approach is also valid for synchronization of fractional-order chaotic systems with the same system structure. One constructive example is given to verify the feasibility and superiority of the proposed method.

In the paper titled "Positive Solutions for a Fractional Boundary Value Problem with a Perturbation Term", the author obtains some new upper and lower estimates for the Green's function associated with a fractional boundary value problem with a perturbation term. Criteria for the existence of positive solutions of the problem are then obtained based on these theories.

In the paper titled "The Exact Iterative Solution of Fractional Differential Equation with Nonlocal Boundary Value Conditions", the authors deal with a singular nonlocal fractional differential equation with Riemann-Stieltjes integral conditions. The exact iterative solution is established under the iterative technique. The iterative sequences have been proved to converge uniformly to the exact solution, and estimation of the approximation error and the convergence rate have been derived. An example is also given to demonstrate the results

In the paper titled "Fixed-Point Theorems for Systems of Operator Equations and Their Applications to the Fractional Differential Equations", the authors study the existence and uniqueness of positive solution for a class of nonlinear binary operator equations systems by means of the cone theory and monotone iterative technique, under more general conditions. The iterative sequence of the solution and the error estimation of the system are given. The authors also use this new result to study the existence and uniqueness of the solutions for fractional differential equations systems involving integral boundary value conditions in ordered Banach spaces as an application.

In the paper titled "Generalization of Hermite-Hadamard Type Inequalities via Conformable Fractional Integrals", the authors establish a Hermite-Hadamard type identity and several new Hermite-Hadamard type inequalities for conformable fractional integrals and present their applications to special bivariate means.

In the paper titled "Fixed Point Theorems for Generalized $\alpha s-\psi$-Contractions with Applications", the authors study the sufficient conditions for the existence of a unique common fixed point of generalized $\alpha s-\psi$-Geraghty contractions in an $\alpha s$-complete partial $b$-metric space. An example in support of the findings is given.

In the paper titled "A New Sufficient Condition for Checking the Robust Stabilization of Uncertain Descriptor Fractional-Order Systems", the authors consider the robust asymptotical stabilization of uncertain class of descriptor fractional-order systems. In the state matrix, the authors require that the parameter uncertainties are time-invariant and norm-bounded. A sufficient condition for the system with the fractional-order $\alpha$ satisfying $1 \leqslant \alpha<2$ in terms of linear matrix inequalities is derived. The condition of the proposed stability criterion for fractional-order system is easy to be verified. An illustrative example is given to show that the result is effective.

In the paper titled "Positive Solutions for a System of Semipositone Fractional Difference Boundary Value Problems", by using the fixed point index, the authors establish two existence theorems for positive solutions to a system of semipositone fractional difference boundary value problems. Nonnegative concave functions and nonnegative matrices to characterize the coupling behavior of our nonlinear terms are adopted.

In the paper titled "Non-Nehari Manifold Method for Fractional p-Laplacian Equation with a Sign-Changing Nonlinearity", the authors consider a class of fractional pLaplacian equation. The nonlinear term $f$ has the subcritical growth and may change sign. Under the condition that $V$ is coercive, the existence of ground state solutions for $\mathrm{p}$ Laplacian equation is established.

In the paper titled " $C *$-Algebra-Valued G-Metric Spaces and Related Fixed-Point Theorems", the authors introduce the notion of the $C *$-algebra-valued $G$-metric space. The existence and uniqueness of some fixed-point theorems for self-mappings with contractive or expansive conditions on complete $C *$-algebra-valued $G$-metric spaces are established. As an application, the authors prove the existence and uniqueness of the solution of a type of differential equations.

In the paper titled "The Tensor Padé-Type Approximant with Application in Computing Tensor Exponential Function", tensor Padé-type approximant is defined by introducing a generalized linear functional for the first time. The expression of TPTA is provided with the generating function form. Moreover, by means of formal orthogonal polynomials, the authors propose an efficient algorithm for computing 
TPTA. As an application, the TPTA for computing the tensor exponential function is presented and some numerical examples are given to demonstrate the efficiency of the proposed algorithm.

In the paper titled "A New Approach to the Existence of Quasiperiodic Solutions for Second-Order Asymmetric $p$-Laplacian Differential Equations", the authors propose a new estimate approach to study the existence of AubryMather sets and quasiperiodic solutions for the second-order asymmetric $p$-Laplacian differential equations. By using the Aubry-Mather theorem, the existence of Aubry-Mather sets and quasiperiodic solutions under some reasonable conditions are obtained. Particularly, the advantage of the approach is that it not only gives a simpler estimation procedure, but also weakens the smoothness assumption on the function $\psi(t, x)$ in the existing literature.

Through the special issue, we also hope to open the opportunity for the journal readers to make comments on the work presented.

\section{Conflicts of Interest}

The editors declare that they have no conflicts of interest regarding the publication of this special issue.

\section{Acknowledgments}

We would like to highly appraise the excellent works of all authors and reviewers.

Xinguang Zhang

Lishan Liu

Yonghong $W u$

Liguang Wang 


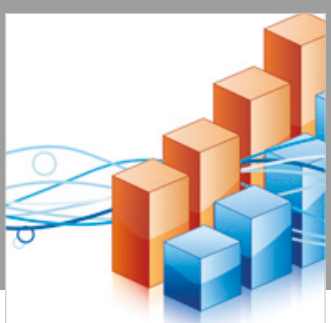

Advances in

Operations Research

\section{-n-m}
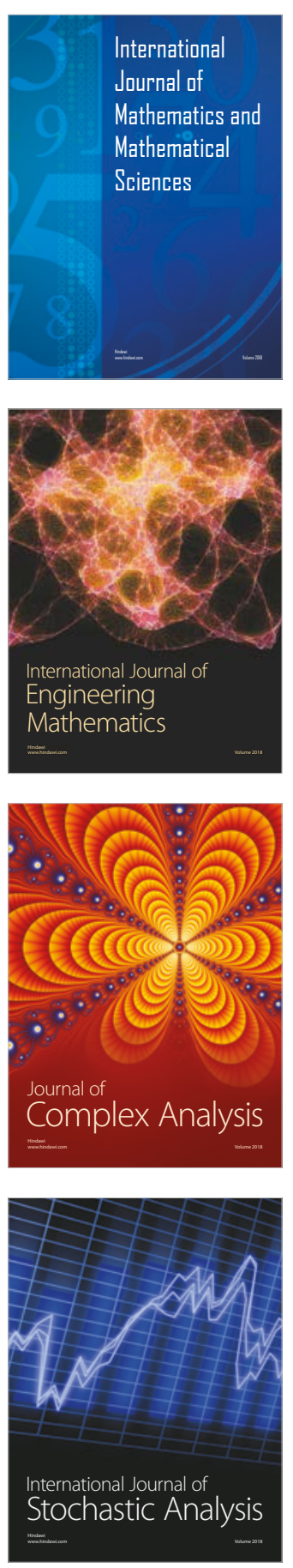
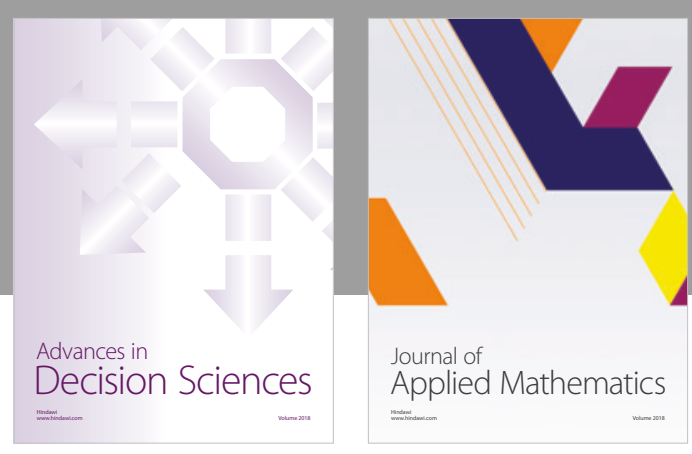

Journal of

Applied Mathematics
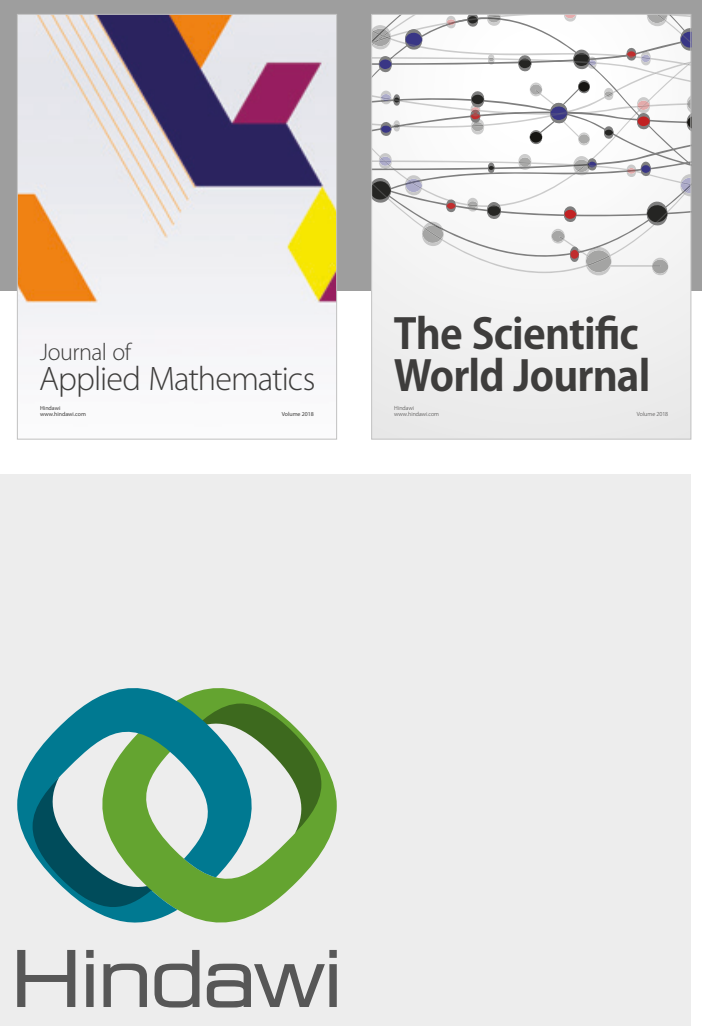

Submit your manuscripts at

www.hindawi.com

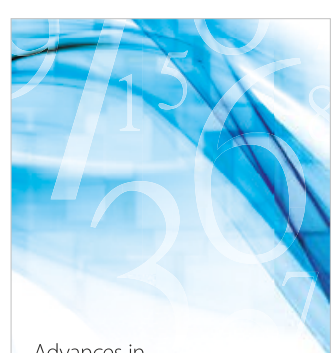

Advances in
Numerical Analysis
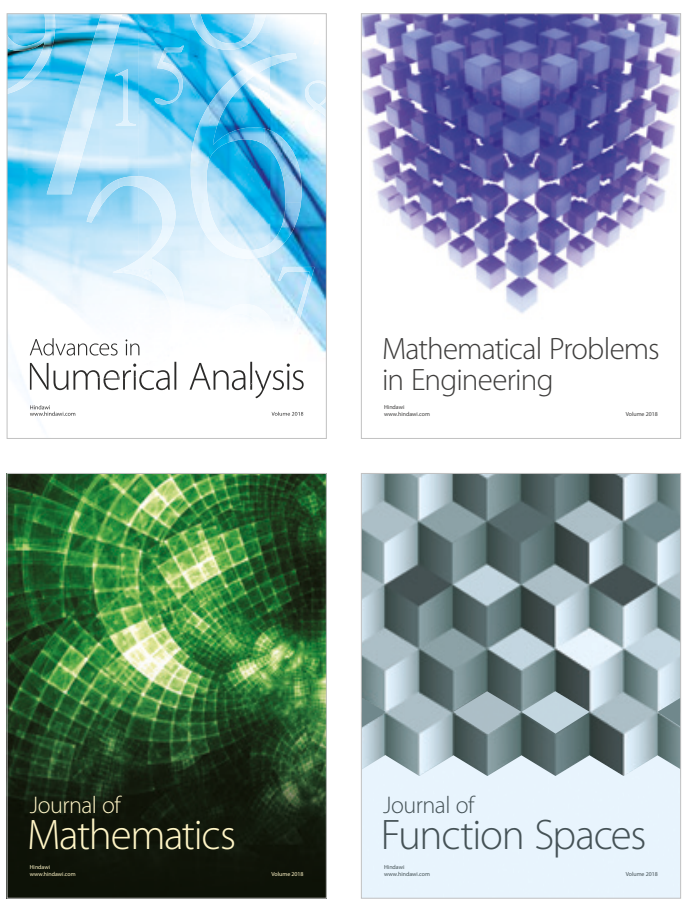

Mathematical Problems in Engineering

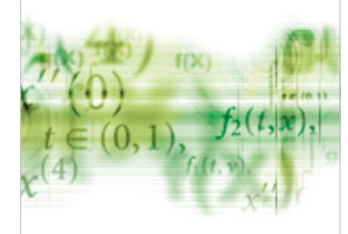

International Journal of

Differential Equations

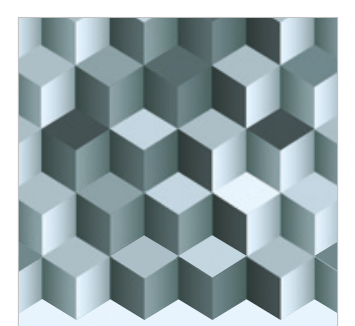

Journal of

Function Spaces

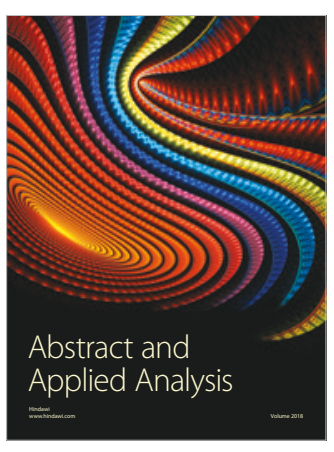

The Scientific

World Journal

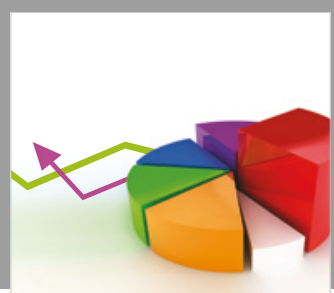

Journal of

Probability and Statistics
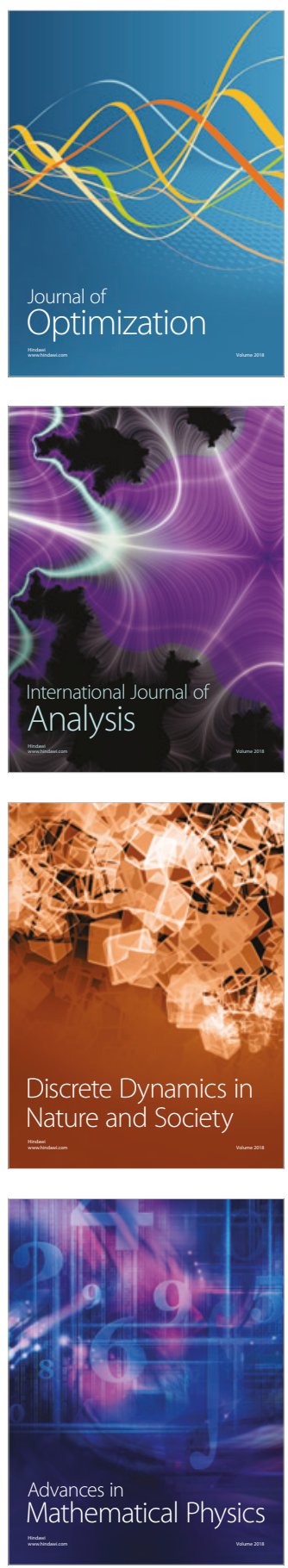